\title{
Phase Identification by Image Processing of EBSD Patterns
}

\author{
E. J. Payton, L. Agudo Jácome, and G. Nolze
}

Federal Institute for Materials Research and Testing (BAM), Division 5.1, Berlin, Germany

Automated electron backscatter diffraction (EBSD) is generally unable to distinguish between multiple cubic phases in a specimen without additional information, such as that obtained by simultaneous energy dispersive X-ray spectroscopy (EDS). Small particles of phases with relatively similar compositions push the limits of phase identification using simultaneous EBSD and EDS, and a mismatch exists between the spatial resolutions of these two techniques due to them having different electron interaction volumes. In a recent paper [1], the present authors explored using backscatter detectors mounted on top of the EBSD detector to obtain atomic number $(Z)$ contrast images that could be used for phase segmentation in cases where the results from the EBSD and EDS signals remain ambiguous. In the present work, we show that similar information can be obtained from the raw EBSD patterns themselves (as previously reported by Wells [2]) at higher spatial resolution than was obtained from the backscatter detectors, with the additional advantage of having no spatial mismatch between the data collection grids.

Processing of the patterns is performed using open-source computational tools developed in-house at the Federal Institute for Materials Research and Testing (BAM), consisting mostly of modules written in Python [3] using the Spyder integrated development environment (IDE) [4]. All EBSD data is being collected using a Bruker system (Bruker Nano GmbH, Berlin, Germany). The Python language was chosen for many reasons, foremost being its ability to easily interface with external dynamic-link libraries written in other programming languages and the reduction in development time that was achievable by being able to import various functions in different namespaces from a variety of freely available add-on packages. The Python(x,y) distribution and Spyder IDE were chosen because they made external collaborations easier by being free, open-source, available on multiple platforms, and having a Matlab-like interface that is relatively easy to learn. The pattern processing module used in the present work integrates seamlessly with the other EBSD analysis tools also being developed by the authors, including modules for analyzing orientation relationships [5] and visualization of EBSD data.

Figure 1 (a) and (b) show typical EBSD patterns from the gamma and gamma prime phases, respectively, from a sample of monocrystalline Ni-based superalloy LEK94 oriented such that (001) is normal to the sample surface. Both phases are cubic, and they exhibit a coherent cube-on-cube orientation relationship with a small lattice misfit. Thus, the patterns cannot be distinguished from one another after background subtraction. However, a systematic difference exists between the intensities observed in the raw patterns, as demonstrated in subfigures (c), (d), and (e). Fig. 1 (c) maps the summed intensity of the first row of pixels of each of the patterns in the EBSD scan. Fig. 1 (d) is formed similarly, but by using only the last row of pixels in the pattern images, while Fig. 1 (e) maps the summed intensity over the entire EBSD detector for each point in the scan. Subfigures (c) and (d) may be thought of as backscatter images formed with the detector at different angular positions relative to the incoming beam, while subfigure (e) can be thought of as a backscatter image formed with a rather large solid angle. The relative signal intensities for the gamma and gamma prime particles invert from the top to the bottom of the detector, as can be seen by comparing (c) to (d). Though the gamma phase has a higher average atomic number by about 2 (which should produce an approximately 5-6\% larger backscatter coefficient by the Reuter model [5]), the gamma prime phase appears systematically brighter 
in the first row images (Fig. 1 (c)) by up to about $9 \%$. The contrast interpretation is complicated by sample tilt and by the surface topography that develops during sample preparation by electropolishing. The magnitude of the surface topography is most clearly gauged from a secondary electron image, as shown in Fig. 1 (f). The computational tools being developed at BAM for the processing of the raw EBSD patterns are being used in combination with Monte Carlo electron trajectory simulations to investigate the effect of surface topography on the backscatter electron signal as well as differences between "channeling in" and "channeling out" behavior in the formation of EBSD patterns.

\section{References:}

[1] E.J. Payton, G. Nolze, Microsc. Microanal. in press (2013).

[2] O.C. Wells et al., Scanning 28 (2006), p. 27.

[3] G. van Rossum et al., "The Python Language Reference", (Python Software Foundation, URL:http://www.python.org), 2013.

[4] P. Raybaut, "Pythonxy: Scientific-oriented Python Distribution Based on Qt and Spyder", (URL:https://code.google.com/p/spyderlib), 2013.

[5] E.J. Payton, A. Aghajani, F. Otto, G. Eggeler, V.A. Yardley, Scripta Mater 66 (2012) 1045.

[6] J. Goldstein, et al., Eds., "Scanning Electron Microscopy and X-Ray Microanalysis", 3rd ed., (Kluwer Academic/Plenum Publishers, New York, NY, USA), 2007.
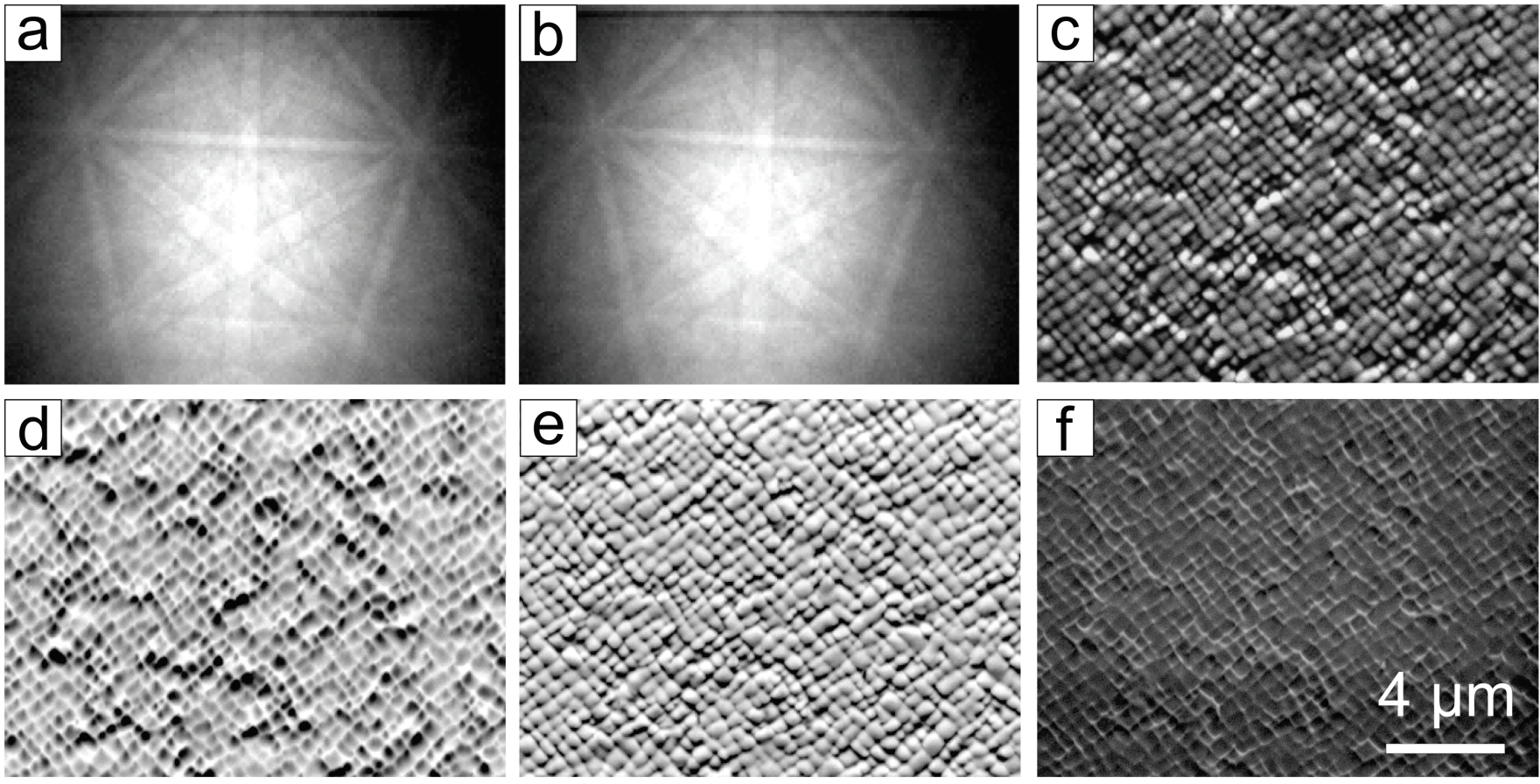

Figure 1. Identifying the gamma and gamma prime phases in monocrystalline Ni-based superalloy LEK94 by processing raw EBSD patterns: (a) typical EBSD pattern for the gamma phase; (b) typical EBSD pattern for the gamma prime phase; (c) map of the summed intensities of the first row of pixels of the raw patterns in an EBSD scan; (d) map of the summed intensities of the last row of pixels; (e) map of the total intensity on the EBSD detector; (f) secondary electron image of the same region, showing magnitude of topography for comparison. The contrast of subfigures (a) and (b) has been enhanced by exactly the same function; the contrast in subfigures (c)-(f) has been enhanced for each subfigure individually by histogram stretching. Subfigures (c)-(f) are on the same scale. 